\title{
DIELECTRIC ROD SURFACE MOUNT ANTENNAS FOR TELECOMMUNICATIONS
}

\author{
S.V.Bukharov, U.V.Gornyak, D.M.Svynarenko, L.Z.Tsypko, L.A.Filins'kyy \\ Oles Honchar Dnipro National University, Dnipro, Ukraine \\ e-mail: sergeyvbuharov@gmail.com
}

\begin{abstract}
The results of modeling broadband antennas for surface mounting on metal surfaces are presented. The considered antennas are a broadband excitation node in a dielectric shell in the form of a fragment of a conical waveguide. The simulation was carried out by the method of moments in the FEKO software environment. The frequency dependences of the input resistance, the standing wave coefficient, and the directional pattern are obtained. Comparison of the modeling results of the excitation node and the results of VSWR measurements of the manufactured model are presented. Modifications of antennas with the direction of the dielectric waveguide along the surface and perpendicular to it are considered. The received antennas can be used by telecommunication systems for installation on mobile and stationary objects.

Keywords: broadband antennas, excitation unit, method of moments, FEKO, frequency dependence of
\end{abstract} VSWR

Received 06.11.2018; Received in revised form 09.12.2018; Accepted 21.12.2018

\section{Introduction}

The antennas used on aircraft and rockets mast take in to account a large number of requirements. One of the requirements is low aerodynamic resistance and mechanical strength. Often antennas are installed with dielectric fairings and this leads to a change in the parameters of the antennas.

Recently, the requirements for on-board data transmission systems on the volume of transmitted information are increasing, which leads to the need to develop new technical solutions in the field of antennas. The requirement of working in a wide frequency band contradicts the requirements of electromagnetic compatibility of systems for various purposes. Therefore, when choosing the type of radiator, a certain compromise is accepted.

Dielectric rod antennas are widely used from centimeter to millimeter wavelengths. They are used as an independent radiator and as an element of the antenna array. The range properties of a dielectric rod antenna are determined by the type of excitation device and the shape of the rod. The rod can be excited by elements of metal waveguides, dipoles, Vivaldi elements $[1,2]$. The rod can be of simple shape, conical, have a modulation of shape along the length or consist of several layers $[3,4]$.

To obtain the required shape of the radiation pattern and the input resistance in the development of such antennas, it is necessary to solve the analysis problems many times. To model the 3D radiating structures, we use the method of moments with the software environment FEKO [5].

\section{The excitation node}

At the first step of the antenna creation, the design of the excitation node is selected and its range properties are specified. Consider the excitation element in the form of a broadband loop monopole with coaxial power (Fig.1a).

The monopole is made of a thin triangular metal plate, which bends and shorts on the screen. Thus, for an element with parameters $a=34 \mathrm{~mm}, b=34 \mathrm{~mm}, h=20 \mathrm{~mm}$ were obtained the frequency dependence of the VSWR (Fig. 1b). Size of the metal screen is $120 \times 300 \mathrm{~mm}$. Practical result of the VSWR measuring is shown on fig.2.

In Figure 1,b, it can be seen that the width of the antenna's operating range by the VSWR level of less than 1.5 is about $300 \mathrm{MHz}$, which is more than enough for any telemetry and control channels as well as the transmission of several video signals. 


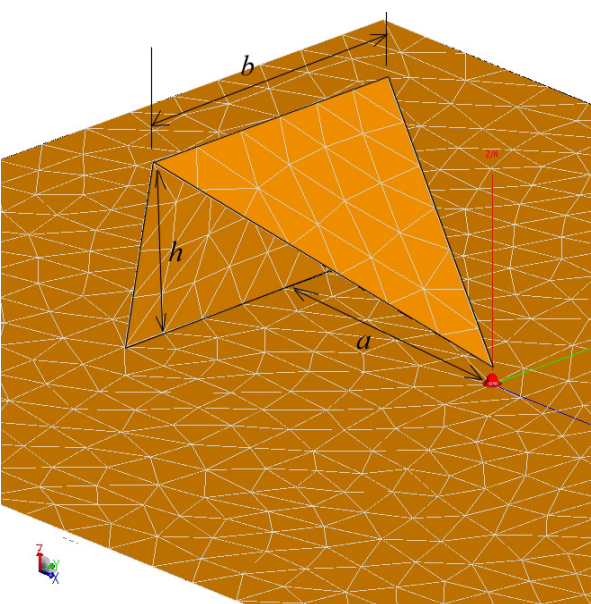

(a)

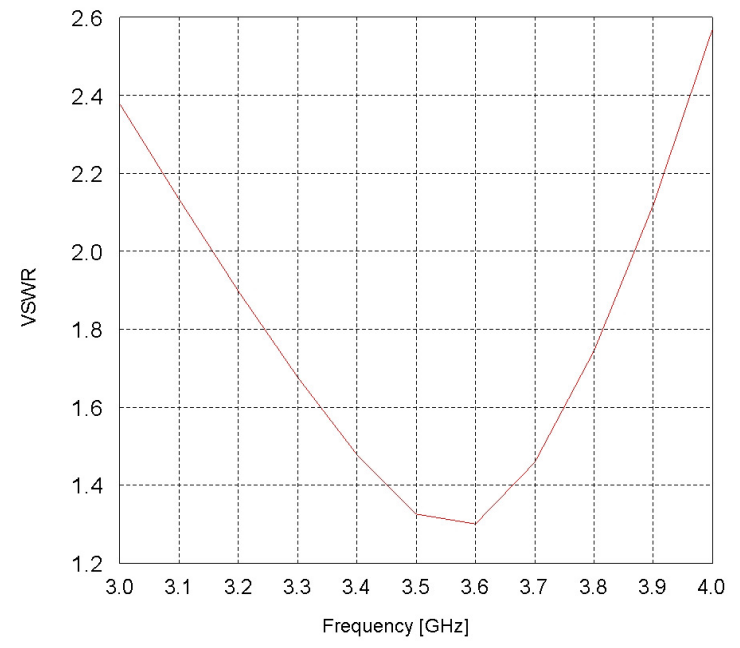

(b)

Fig.1 The wideband excitation node, the parameters of the model (a), the VSWR vs frequency dependence (b).

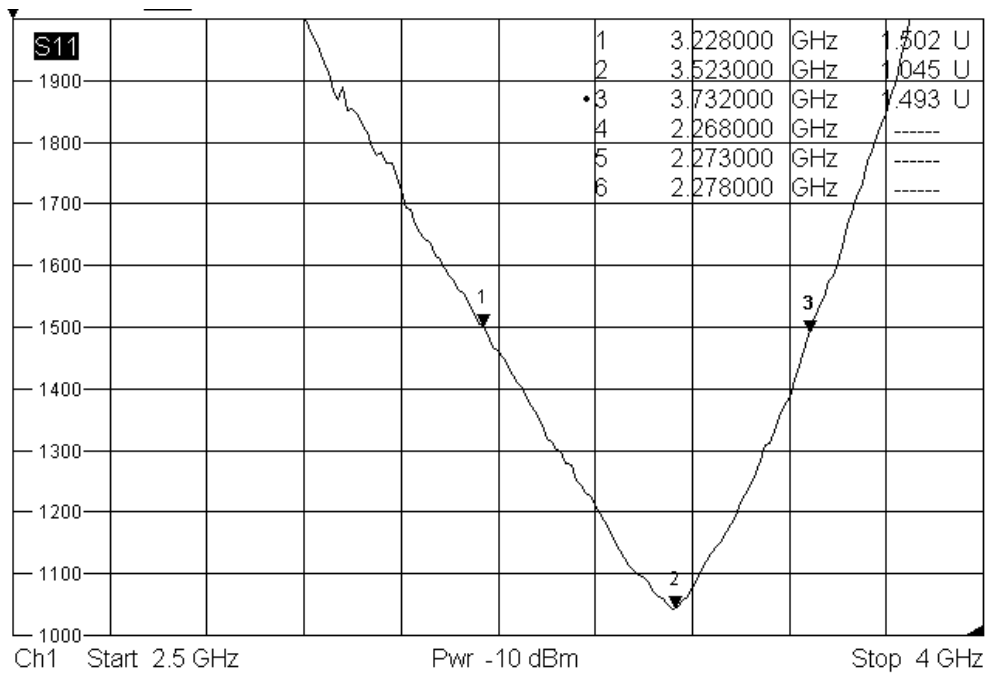

Fig.2 The measured VSWR vs frequency dependence for excitation node.

The directivity pattern of the excitation node is directed upward at some angle to the normal and has a gain of about $5.6 \mathrm{~dB}$ at the maximum.

\section{Results of the surface mount antennas modeling}

In order to give the antenna more directional properties, the excitation unit is immersed in a liquid dielectric, which after solidification forms a dielectric waveguide on the surface of the metal. Depending on the magnitude of the dielectric constant, it is necessary to optimize the dimensions of the excitation unit for reaching the required frequency range.

Figure 3a shows a model for the elliptic shape of a dielectric waveguide (green color). The length of the rod fragment is $250 \mathrm{~mm}$, the width is $50 \mathrm{~mm}$, the height at the beginning of the waveguide is $30 \mathrm{~mm}$ and the dielectric constant of the material is 2.5 . Figure $3 \mathrm{~b}$ shows another excitation node in the dielectric cone fragment. Figure 4 shows the modeling results for different types of antennas. 


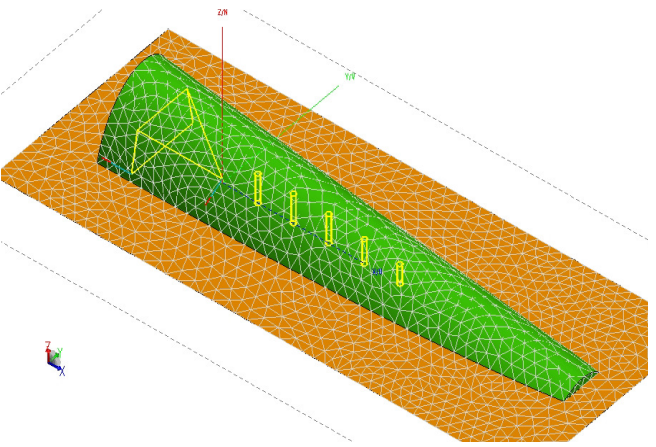

(a)

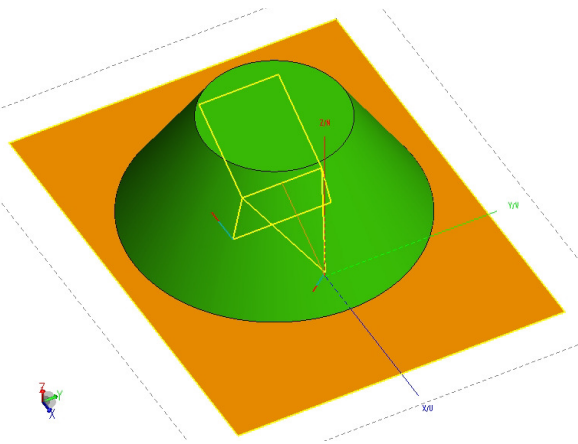

(b)

Fig.3 Surface mount antennas. Antenna with directors (a) forming radiation along the rod. The dielectric conic antenna (b) forming radiation in the perpendicular to the surface direction.

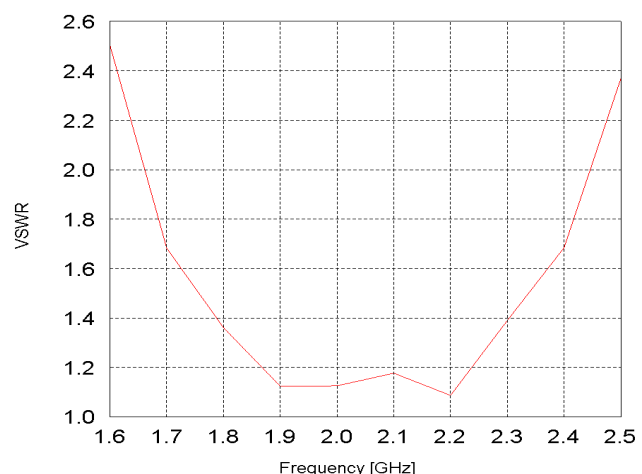

(a)

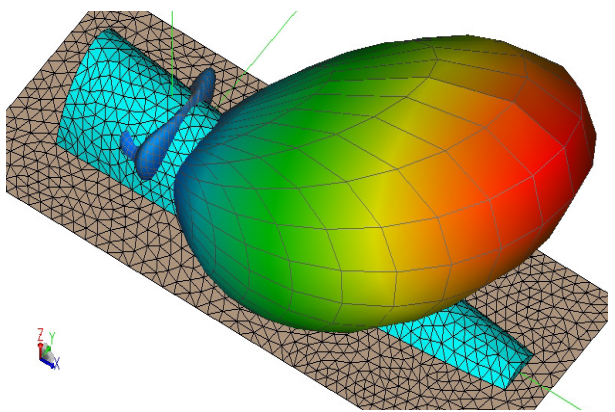

(c)

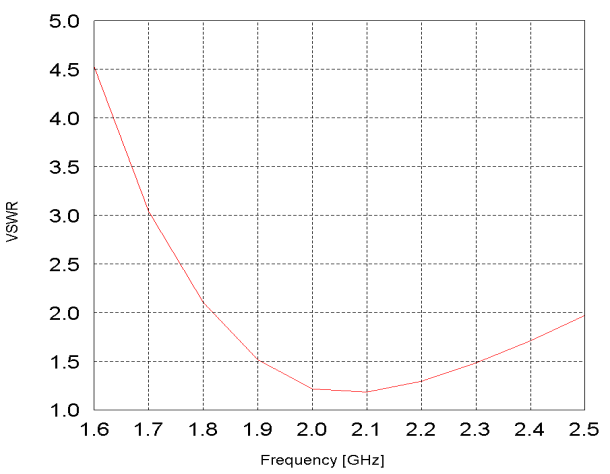

(b)

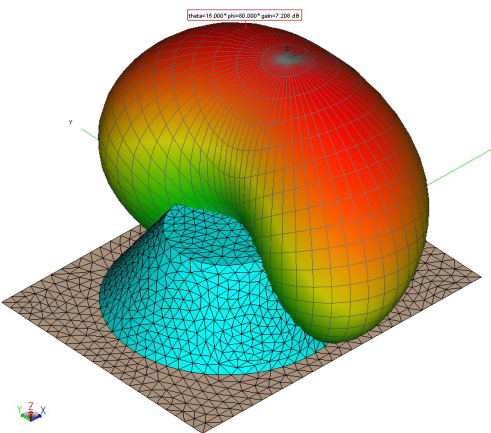

(d)

Fig.4 Results of the modeling for different types of antennas. The VSWR vs frequency dependences are: (a) and (b). Directional patterns at $2000 \mathrm{MHz}$ : (c) -gain in maximum is above $10 \mathrm{~dB}$, beam width by level 0.5 is above 30 degrees and (d) - gain in maximum is above $7 \mathrm{~dB}$, beam width in $\mathrm{ZX}$ plane is above 40 degrees, in $\mathrm{ZY}$ is 130 degrees.

To enhance the directional properties or control the beam, single emitters are combined into arrays (Fig.5). So, for example, for four elements with a one phase excitation, the radiation pattern will have the form shown in the fig.5a.

The permittivity of the dielectric material can vary with increasing frequency, model does not takes in to account thickness of the monopole, so the model is approximate. This design is not optimal and can be improved by changing all parameters of model: excitation node, quantity and sizes of directors, permittivity and shape of the fairing. 


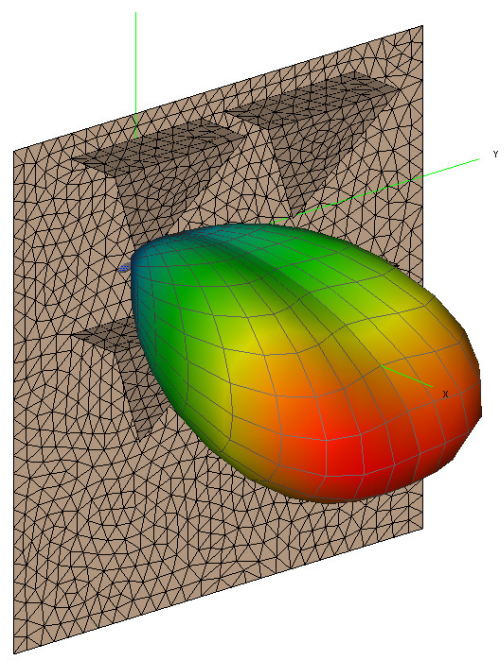

(a)

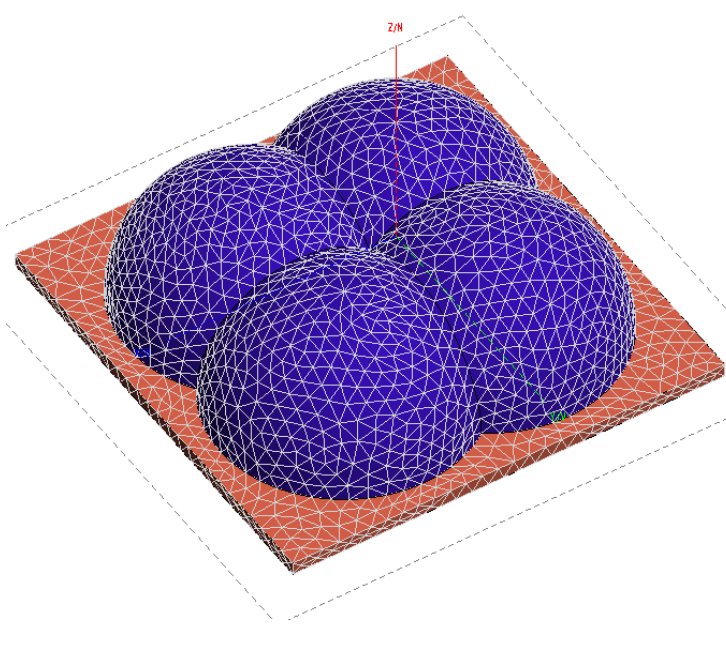

(b)

Fig.5 Antenna arrays. Lattice without fairing, directivity is about $10 \mathrm{~dB}$. Grid with a fairing in the form of dielectric lenses (b), dielectric lenses increase the directivity up to $12 \mathrm{~dB}$.

\section{Conclusions}

The presented antennas can be used as an independent antenna and as a part of the grid. The presented examples can be applied in radio monitoring, some radar applications, in telecommunication systems.

\section{References}

1. Jeremie, Bourqui Balanced antipodal Vivaldi antenna with dielectric director for near-field microwave imaging / B. Jeremie, M. Okoniewski, E. C. Fear // IEEE Transactions on Antennas and Propagation. - 2010. - Vol. 58, No. 7. - P. $2318-2326$

2. Herzi, Rabiaa Enhanced radiation characteristics for antipodal Vivaldi antenna using high permittivity dielectric director / Rabiaa Herzi, Moufida Bouslama, Ali Gharsallah // Journal of advanced dielectrics. - 2017. - Vol. 7, No. 3. DOI: 10.1142/S2010135X17500187 3. Lin, Song Dielectric rod antennas array fed using substrate integrated waveguides / Lin, Song, et al // Antennas and Propagation Society International Symposium. 2008. AP-S 2008. IEEE, 2008. DOI: 10.1109/APS.2008.4618994.

4. Hanham, S. A ring slot excited dielectric rod antenna for terahertz imaging / S. Hanham, T.S. Bird, B. Johnston // IEEE AP-S Int. Symp., Honolulu, HI, Jul. 10 - 15, 2007, P. $5539-5542$.

5. Bankov, S. Evaluation of radiated structures using FEKO / S. Bankov, A. Kurushin. - Moscow, ZAO NPP "RODNIK" 2008, 246 p. (in russian) 